\title{
Photospheric supergranular flows and magnetic flux emergence
}

\author{
M. Stangalini
}

\author{
INAF - Osservatorio Astronomico di Roma, 00040 Monte Porzio Catone (RM), Italy \\ e-mail: marco.stangalini@inaf.it
}

Received 11 October 2013 / Accepted 9 December 2013

\section{ABSTRACT}

\begin{abstract}
A recent study carried out on high-sensitivity SUNRISE/IMAX data has reported about areas of limited flux emergence in the quiet Sun. By exploiting an independent and longer (four hours) data set acquired by Hinode/SOT, we investigate these regions in more detail by analysing their spatial distribution and relation with the supergranular flow. Our findings, while confirming these calm areas, also show that the emergence rate of small magnetic elements is largely suppressed at the locations where the divergence of the supergranular plasma flows is positive. This means that the dead-calm areas previously reported in literature are not randomly distributed across the solar photosphere, but are linked to the supergranular cells themselves. These results are discussed in the framework of the recent literature.
\end{abstract}

Key words. Sun: magnetic fields - Sun: photosphere

\section{Introduction}

Recently, Martínez González et al. (2012b) have identified areas of reduced flux emergence in the quiet photosphere on spatial scales larger than granules $(\sim 1000 \mathrm{~km})$, which they named deadcalm areas. These areas also show a moderate deficit of circular polarization signals in the deep magnetograms obtained from the same data by Martínez Pillet (2013).

It has been found that small magnetic elements with diameters comparable to or below the present resolving power of the current solar telescopes $(\sim 100-150 \mathrm{~km}$ on the solar surface) are abundant in the very quiet Sun areas of the solar photosphere (Orozco Suárez et al. 2007; Lites et al. 2008; Viticchié 2012; Martínez González et al. 2012a). They have been observed to emerge in small magnetic loops within supergranules (e.g. Centeno et al. 2007; Martínez González \& Bellot Rubio 2009) and then diffuse radially toward the edges of the supergranules themselves (Abramenko et al. 2011; Orozco Suárez et al. 2012; Lepreti et al. 2012; Giannattasio et al. 2013), to form the socalled magnetic network (Simon \& Weiss 1989). In particular, by exploiting a very long series of high-resolution magnetograms (height hours) Orozco Suárez et al. (2012) have observed the small magnetic concentrations within a supergranule moving radially outward from the centre of the supergranule toward the network, with a velocity aligned to the plasma flow. Their results have also shown that the dynamical properties of these magnetic concentrations depend on their location within the supergranule itself. They indeed accelerate and then decelerate in the proximity of the supergranular boundaries. The same authors have argued that this may affect the spatial distribution of the magnetic flux observed, because the magnetic diffusion is more effective in some areas of the supergranular cells. In this regard, Giannattasio et al. (2013) have observed a change of the diffusion regime of small magnetic elements with a reduction of the diffusivity at spatial scales larger than granulation and comparable with supergranular scales.
Small magnetic elements in the solar photosphere are also thought to be of some importance in deciphering different unsolved question in solar physics, for example the heating of the upper layers of the Sun's atmosphere through the excitation of MHD waves (see for example Martínez González et al. 2011; Jafarzadeh et al. 2013; Stangalini et al. 2013b,a) and their contribution to the solar irradiance (Rast \& Harder 2012). Although, as seen before, they have been studied in detail in many aspects, their origin is still a matter of debate. Indeed, two main competing theories have been put forward to explain these small scale magnetic fields in the solar photosphere. In the first scenario these magnetic fields are the result of the decay of larger active regions produced by the global dynamo. In the second scenario the magnetic elements are generated by a local small-scale dynamo (SSD) driven by granular and supergranular plasma flows. Both mechanisms are likely to contribute at the same time, although it has to be determined which is the dominant one (Cattaneo \& Hughes 2001).

However, in the past few years, many observational results have been put forward in favour of the SSD (e.g. Ishikawa et al. 2008; Danilovic et al. 2010a; Lites 2011; Buehler et al. 2013), as well as results from numerical simulations of the solar convective zone (Vögler \& Schüssler 2007; Schüssler \& Vögler 2008; Pietarila Graham et al. 2010; Danilovic et al. $2010 b)$. For a deeper examination of the observational evidence in favour of the SSD we refer to the review by Martínez Pillet (2013). It is unclear, however, how these calm areas observed by Martínez González et al. (2012b) might fit into this general framework, although they represent an important constraint on the generation and amplification of the magnetic field on small scales in the solar photosphere. Among the many possible explanations for the existence of these areas, one interesting possibility, indicated by the authors themselves, is that this might be due to the highly intermittent character of the SSD (Cattaneo \& Hughes 2001), which leads to a bursty appearance of smallscale magnetic fields. Moreover, Tobias \& Cattaneo (2008) have demonstrated that coherent flows with their sustained stretching 
are far more efficient, in terms of dynamo action, than incoherent plasma flows. For this reason the same authors argued that the role of coherent structures should always be carefully assessed when dealing with SSDs.

The IMAX data analysed by Martínez González et al. (2012b) had a limited temporal duration ( 30 min). This prevented a possible analysis of the calm areas in relation to largescale (tens of $\mathrm{Mm}$ ) plasma flows such as those that determine the supergranular patterns. In this study we take advantage of the long temporal duration of the data provided by SOT/FG, the narrow-band imager on board the Hinode satellite (Tsuneta et al. 2008), to investigate these areas in more detail and to assess the role of supergranular flows in their origin.

\section{Data set and analysis}

The data set used in this study consists of a sequence of high spatial resolution magnetograms and intensity measurements acquired by SOT/NFI in the Na I $589.6 \mathrm{~nm}$ spectral line that is sampled at two wavelength positions from the line centre ( $\pm 160 \mathrm{m \AA})$. The magnetograms were generated from shutterless $V$ and $I$ Stokes filtergrams taken on 2008 August 18 close to the disc centre. The temporal cadence of the data is $30 \mathrm{~s}$. The data are slightly downsampled because the pixel scale was set at 0.16 arcsec, while the diffraction limit was $\sim 0.24$ arcsec. The field of view $(\mathrm{FoV})$ was approximately $80 \times 80$ arcsec, and the total duration of the time sequence was approximately four hours. In Fig. 1 (panel a) the time-averaged magnetogram saturated between $-50 \mathrm{G}$ and $50 \mathrm{G}$ is shown. The FoV encompasses a few supergranules whose boundaries are highlighted by the network patches visible in the figure.

In addition to the standard calibration procedure (the IDL code $f g$-prep.pro available in the Hinode Solarsoft package), a registration procedure allowing subpixel accuracy was applied. This was done to account for any possible residual misalignment caused by satellite jitter and tracking errors. This procedure is based on a fast Fourier transform (FFT) cross-correlation and used the whole FoV to estimate the misalignment between two images. We iteratively applied the FFT registration, until the mean residuals were minimized (until a stable value was reached). This occurred after three iterations.

To study the emergence of small magnetic features we employed the YAFTA tracking algorithm (Welsch \& Longcope 2003; DeForest et al. 2007), which allowed us to identify and track magnetic pixels belonging to the same local maximum. For tracking purposes two constraints were applied. Only features lying above a threshold in the magnetograms and with an area as small as the sampling resolution limit set by the pixel scale were considered $(2 \times 2$ pixels in our case, corresponding to $240 \times 240 \mathrm{~km}$ in the solar photosphere). The threshold on the magnetic signal was chosen to be $2 \sigma$ (equivalent to $11.8 \mathrm{G}$ ). Following Liu et al. (2012), we estimated the sigma of the magnetic signal by fitting a Gaussian to the low-field pixels with an absolute value of the magnetic flux density below $200 \mathrm{G}$. With the YAFTA tracking code, we identified the newly appeared magnetic elements and studied their initial position in the FoV. From the complete sample of newly appeared magnetic elements that fulfilled the above criteria, we only retained those lasting (trackable) at least three temporal steps ( $\sim 90 \mathrm{~s})$. This was done to avoid spurious detections. Lamb et al. (2010) have shown that up to of $75 \%$ of the newly appeared magnetic elements in magnetograms can be ascribed to coalescence, in which a magnetic flux tube undergoes a field intensification, thus becoming detectable. This makes the detection of emergence events difficult. Since

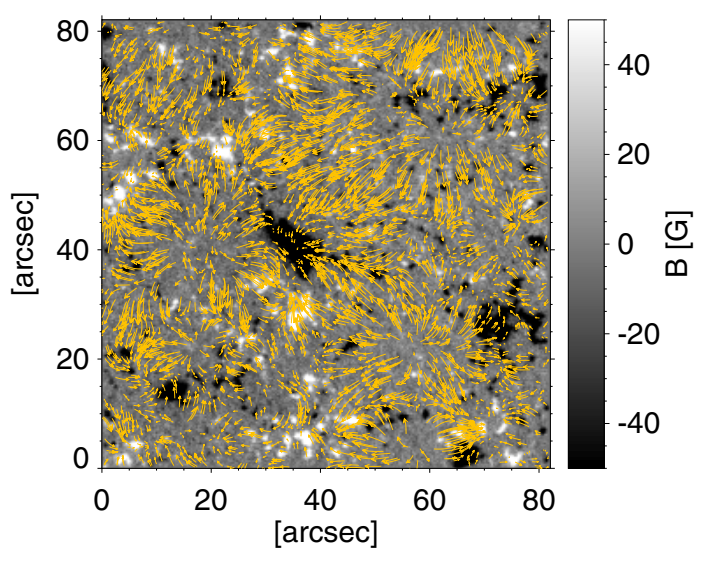

(a) Horizontal velocity field

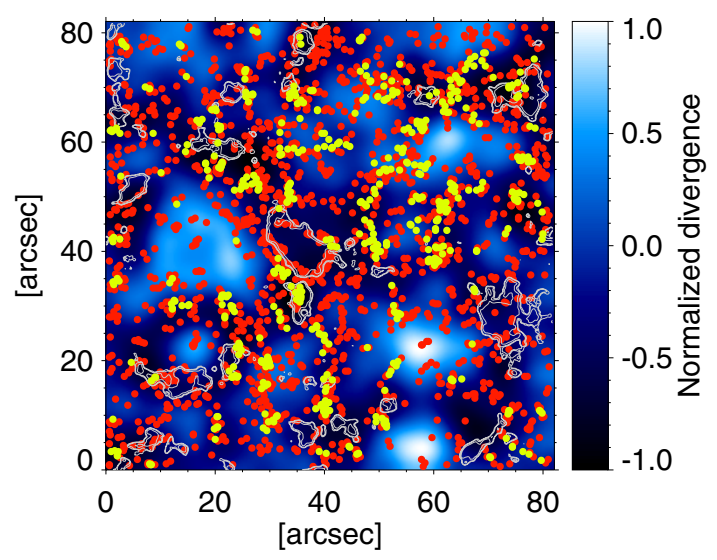

(b) Map of divergence and flux emergence

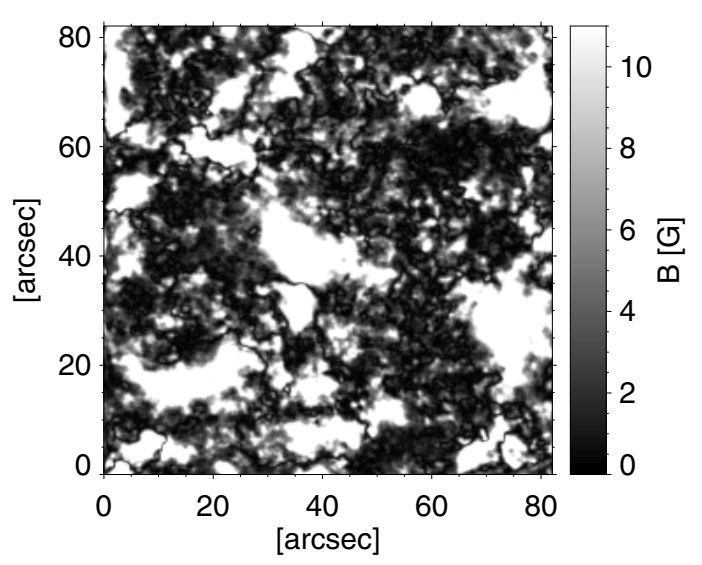

(c) Deep magnetogram

Fig. 1. a) Time-averaged magnetogram obtained by averaging over the total duration of the data set with the horizontal velocity field obtained from the local correlation tracking superimposed (LCT, orange arrows). b) Normalized divergence map obtained from LCT with the location of magnetic appearance events over the whole duration of the data set (red dots), and flux emergence events (yellow dots). The white contours indicate the location of the maxima of the absolute value of the magnetic field $(|B|>50 \mathrm{G})$. c) Deep absolute magnetogram saturated at $11 \mathrm{G}$.

the tracking algorithm does not distinguish between different physical processes that lead to the appearance of new magnetic features, we used the tracked bipolar and cluster method (hereafter TBCM) developed and used in Close et al. (2005a,b) and 
Thornton \& Parnell (2011) to identify emergences. This method, starting from the tracked elements and using the birth information provided by the tracking code, identifies newly emerged bipoles and clusters and selects unique pairings among them. More in detail, the code searches for features that satisfy the following conditions: the distance between two opposite polarity features $D_{0}$ and the temporal difference of appearance $\Delta T$ must be smaller than a fixed quantity, and the ratio of the fluxes $\rho$ must satisfy the relation $1 / \rho \leq\left|\phi_{i+}\right| /\left|\phi_{i-}\right| \leq \rho$, where $\phi_{i+}$ and $\phi_{i-}$ represent the magnetic fluxes associated to the footpoints of the bipole. At this point, not all the emerging bipoles and clusters identified with the TBCM method represent unique feature pairings (emerging loops or biboles). This can be accomplished by using a connectivity matrix as in Close et al. (2005b) and Thornton \& Parnell (2011). Similar to Thornton \& Parnell (2011), we chose $\rho=3, \Delta T=15 \mathrm{~min}$, and $D_{0}=1.3 \mathrm{Mm}$. Our aim was assessing possible effects of supergranular flows on the spatial distribution of the flux emergence. For this purpose we estimated the supergranular flow field by using a local correlation tracking technique (LCT). In particular, we made use of the fast local correlation tracking code (FLCT, Fisher \& Welsch 2008) with a spatial window of 12 pixels, or equivalently, $\simeq 1400 \mathrm{~km}$ which is slightly larger than the granular scale. The granules are thought to be a good tracers of the large-scale flows. Each intensity image of the sequence is used in the LCT, thus the original temporal cadence is maintained. Finally, the horizontal velocity fields at each time step were used to estimate the average velocity field over the whole duration of the data set ( $\sim$ four hours). The final horizontal velocity field was additionally smoothed with a running window of $10 \times 10$ pixels $(1200 \times 1200 \mathrm{~km})$ to further reduce the granular noise. The final result of this analysis is overplotted in Fig. 1 (panel a, yellow arrows). The FoV encompasses a few supergranules, as clearly indicated by the direction of the horizontal flows.

\section{Results}

We studied the spatial distribution of emergence events, which was derived using the information provided by the tracking algorithm together with the TBCM method described above, and searched for a possible relation with the horizontal velocity fields obtained from the LCT. Figure 1, panel b, shows the position of the newly appeared magnetic elements (red dots), and disambiguated newly emerged bipoles and clusters (yellow dots) obtained from the TBCM code, overplotted on the divergence map obtained from LCT. In agreement with Martínez González et al. (2012b), we found that the magnetic elements emerge non-homogeneously generating areas that lack frequent flux emergence. Moreover, the comparison with the divergence map showed that these areas occupy regions characterized by positive divergence (sources of the supergranular flow field). Panel c of the same figure also depicts the mean deep magnetogram (saturated at $\pm 11 \mathrm{G}$ ), to be compared with the divergence map of panel b. Similar to what was reported by Martínez Pillet (2013), the deep magnetogram also shows a lack of residual magnetic signal at the same locations at which an emergence deficit is seen. Even in this case, it is straightforward to see that these regions correspond to areas with positive divergence. This is even clearer in Fig. 2, where we plot the emergence frequency of the magnetic elements selected with the TBCM method as a function of the divergence. The emergence frequency is lower at the locations of positive divergence (centres of supergranules) and higher at the boundaries of the convective cells (negative divergence).

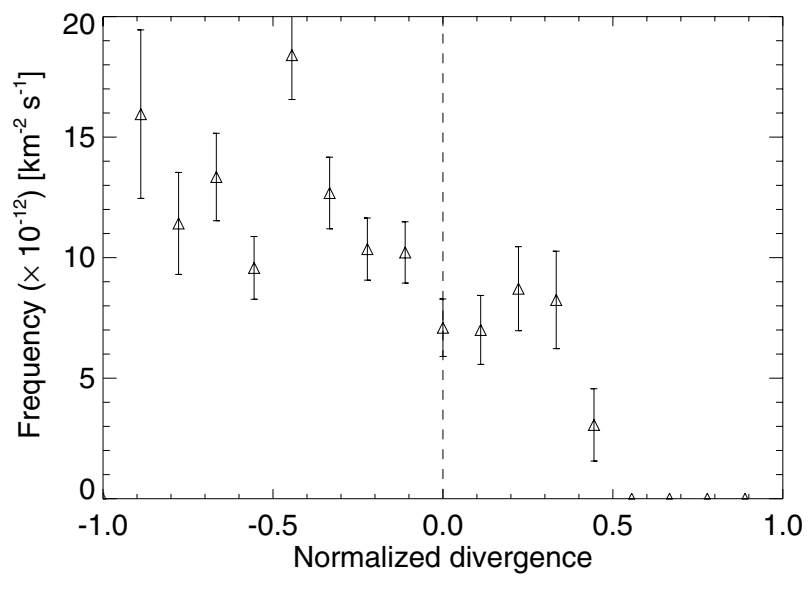

Fig. 2. Flux emergence frequency derived from tracking as a function of the divergence of the flow field estimated from the LCT. The error bars represent the standard error of the mean.

\section{Discussions and conclusions}

By analysing a long series of high-cadence and high spatial resolution magnetograms acquired with SOT/FG, we have studied the spatial distribution of flux emergence in supergranules. In particular, we investigated the flux emergence frequency in relation to the supergranular flows. Very long time-series are indeed required to distinguish the supergranular from granular flows in LCT analyses. From newly appeared magnetic elements provided by the YAFTA tracking code, we selected only the flux emergence cases by means of the TBCM method. As underlined in Thornton \& Parnell (2011), this method relies on strict criteria for selecting emerging bipoles and clusters of magnetic elements. Our results confirm the surprisingly quieter magnetic areas in the solar photosphere, which were previously reported by Martínez González et al. (2012b) from SUNRISE/IMAX data. Our results are also consistent with the findings by Wang (1988), who observed a slight tendency of the magnetic fields to emerge close to the supergranular boundaries. In addition to the results of Martínez González et al. (2012b), we also found that these dead-calm areas coincide with locations of positive divergence of the supergranular plasma flows. We found that the flux emergence rate is indeed maximally reduced at the centres of supergranules, while it increases toward their borders. This behaviour is also seen in deep magnetograms where a lack of residual magnetic signal can be found in correspondence with the centres of supergranules. Although this deficit of residual magnetic signal was already reported by Martínez Pillet (2013), no link with supergranular flows was possible in that case due to the limited duration of the data sets analysed.

Framing these results in an SSD scenario is difficult (Martínez Pillet 2013), but it has been shown that coherent flows and velocity patterns lead to the development of efficient dynamos in comparison with random flows. Indeed, Tobias \& Cattaneo (2008) have demonstrated that the amplification of the magnetic field in an SSD is strongly dependent on spatially and temporally coherent structures. In particular, when two velocity flows with the same spectra are considered, the one with larger spatially and temporally coherent structures results in a more efficient SSD mechanism. In addition, the same authors have shown that coherent velocity flows lead to the generation of less homogeneous magnetic field distributions owing to the line stretching of the coherent flows themselves. 
Although one might be tempted to believe that the granular flows are the most obvious patterns giving birth to the small-scale magnetic fields detected in high-resolution and highsensitivity observations, the supergranulation has been also indicated as a possible mechanism from which a dynamo action may originate (Cattaneo 1999). This, together with the above scenario of enhanced amplification produced by regular flows, may constitute an important ingredient to be considered in explaining our observational findings.

Martínez Pillet (2013) has recently pointed out that the voids of flux emergence observed by Martínez González et al. (2012b) are barely consistent with a granularly driven SSD because they occur on larger scales $(>1 \mathrm{Mm})$. In this connection it is worth noting that Guglielmino et al. (2012) have studied a magnetic bipole that emerged in the solar photosphere and reported a footpoint separation of $\sim 4 \mathrm{Mm}$, which is also larger than the granular scale, hence inconsistent with an SSD driven by granular convection.

Our results also fit this picture by demonstrating a strongly non-homogeneous emergence pattern of the magnetic field across the FoV. This is not surprising in light of the results of Tobias \& Cattaneo (2008) who demonstrated that the process of stretching and amplification of the field through an SSD can be highly non-local and patchy, depending on the properties of the coherent structures of the velocity field. Owing to the finite sensitivity of the instrumentation, we cannot argue that the flux emergence is entirely inhibited at the centre of supergranules. It may well be that the emerging magnetic field generated by an SSD is far from being detectable at that locations, probably because of a less efficient amplification owing to the supergranular pattern and its dynamics. However, our results suggest a relation between the flux emergence rate (and or the amplification of the field) and the supergranular flows.

In this regard, another and perhaps more intriguing explanation for our findings may come from different considerations. As mentioned above, Orozco Suárez et al. (2012) have argued that because the dynamical properties (i.e. the radial velocity) of the magnetic elements depend on their position within the supergranule, the spatial distribution of observed magnetic fields might be affected by this, resulting in an inhomogeneous distribution. This is a direct consequence of the magnetic diffusion being more effective in certain locations.

Acknowledgements. This work has been supported by the PRIN-INAF 2010 grant, funded by the Italian National Institute for Astrophysics (INAF). We thank Francesco Berrilli and Fabio Giannattasio for useful discussions. We also thank Ilaria Ermolli for a critical reading of the first draft of this manuscript and the anonymous referee for providing useful comments and suggestions, which helped a lot to improve the first version of the paper. Hinode is a Japanese mission developed and launched by ISAS/JAXA, collaborating with NAOJ as a domestic partner, NASA and STFC (UK) as international partners. Scientific operation of the Hinode mission is conducted by the Hinode science team organized at ISAS/JAXA. This team mainly consists of scientists from institutes in the partner countries. Support for the post-launch operation is provided by JAXA and NAOJ (Japan), STFC (UK), NASA, ESA, and NSC (Norway).

\section{References}

Abramenko, V. I., Carbone, V., Yurchyshyn, V., et al. 2011, ApJ, 743, 133 Buehler, D., Lagg, A., \& Solanki, S. K. 2013, A\&A, 555, A33

Cattaneo, F. 1999, ApJ, 515, L39

Cattaneo, F., \& Hughes, D. W. 2001, Astron. Geophys., 42, 3.18

Centeno, R., Socas-Navarro, H., Lites, B., et al. 2007, ApJ, 666, L137

Close, R. M., Parnell, C. E., Longcope, D. W., \& Priest, E. R. 2005a, Sol. Phys., 231, 45

Close, R. M., Parnell, C. E., \& Priest, E. R. 2005b, Geophys. Astrophys. Fluid Dyn., 99, 513

Danilovic, S., Beeck, B., Pietarila, A., et al. 2010a, ApJ, 723, L149

Danilovic, S., Schüssler, M., \& Solanki, S. K. 2010b, A\&A, 513, A1

DeForest, C. E., Hagenaar, H. J., Lamb, D. A., Parnell, C. E., \& Welsch, B. T. 2007, ApJ, 666, 576

Fisher, G. H., \& Welsch, B. T. 2008, in Subsurface and Atmospheric Influences on Solar Activity, eds. R. Howe, R. W. Komm, K. S. Balasubramaniam, \& G. J. D. Petrie, ASP Conf. Ser., 383, 373

Giannattasio, F., Del Moro, D., Berrilli, F., et al. 2013, ApJ, 770, L36

Guglielmino, S. L., Martínez Pillet, V., Bonet, J. A., et al. 2012, ApJ, 745, 160

Ishikawa, R., Tsuneta, S., Ichimoto, K., et al. 2008, A\&A, 481, L25

Jafarzadeh, S., Solanki, S. K., Feller, A., et al. 2013, A\&A, 549, A116

Lamb, D. A., DeForest, C. E., Hagenaar, H. J., Parnell, C. E., \& Welsch, B. T. 2010, ApJ, 720, 1405

Lepreti, F., Carbone, V., Abramenko, V. I., et al. 2012, ApJ, 759, L17

Lites, B. W. 2011, ApJ, 737, 52

Lites, B. W., Kubo, M., Socas-Navarro, H., et al. 2008, ApJ, 672, 1237

Liu, Y., Hoeksema, J. T., Scherrer, P. H., et al. 2012, Sol. Phys., 279, 295

Martínez González, M. J., \& Bellot Rubio, L. R. 2009, ApJ, 700, 1391

Martínez González, M. J., Asensio Ramos, A., Manso Sainz, R., et al. 2011, ApJ, 730, L37

Martínez González, M. J., Bellot Rubio, L. R., Solanki, S. K., et al. 2012a, ApJ, 758, L40

Martínez González, M. J., Manso Sainz, R., Asensio Ramos, A., \& Hijano, E. 2012b, ApJ, 755, 175

Martínez Pillet, V. 2013, Space Sci. Rev., 178, 141

Orozco Suárez, D., Bellot Rubio, L. R., del Toro Iniesta, J. C., et al. 2007, ApJ, 670, L61

Orozco Suárez, D., Katsukawa, Y., \& Bellot Rubio, L. R. 2012, ApJ, 758, L38

Pietarila Graham, J., Cameron, R., \& Schüssler, M. 2010, ApJ, 714, 1606

Rast, M. P., \& Harder, J. W. 2012, in Second ATST-EAST Meeting: Magnetic Fields from the Photosphere to the Corona., eds. T. R. Rimmele, A. Tritschler, F. Wöger, et al., ASP Conf. Ser., 463, 65

Schüssler, M., \& Vögler, A. 2008, A\&A, 481, L5

Simon, G. W., \& Weiss, N. O. 1989, ApJ, 345, 1060

Stangalini, M., Berrilli, F., \& Consolini, G. 2013a, A\&A, 559, A88

Stangalini, M., Solanki, S. K., Cameron, R., \& Martínez Pillet, V. 2013b, A\&A, 554, A 115

Thornton, L. M., \& Parnell, C. E. 2011, Sol. Phys., 269, 13

Tobias, S. M., \& Cattaneo, F. 2008, Phys. Rev. Lett., 101, 125003

Tsuneta, S., Ichimoto, K., Katsukawa, Y., et al. 2008, Sol. Phys., 249, 167

Viticchié, B. 2012, ApJ, 747, L36

Vögler, A., \& Schüssler, M. 2007, A\&A, 465, L43

Wang, H. 1988, Sol. Phys., 117, 343

Welsch, B. T., \& Longcope, D. W. 2003, ApJ, 588, 620 